dose reduction; no episodes of sepsis were seen. After 2-4 weeks of combination therapy, mean 6-thioguanine levels had markedly increased and 6-methylmercaptopurine levels correspondingly decreased. Patients with Crohn's disease experienced significantly reduced disease activity $(P=0.001)$ from baseline, and there was a similar trend in those with ulcerative colitis. Combination therapy allowed mean daily prednisone doses to be greatly reduced (from $17.6 \mathrm{mg}$ to $1.8 \mathrm{mg}$ ), and 8 of 16 initially steroid-dependent patients discontinued prednisone entirely. Patients' levels of liver transaminases also normalized, consistent with their reduced 6-methylmercaptopurine levels.

Sparrow and colleagues note that allopurinol and thiopurine combination therapy is only appropriate for IBD patients with adequate leukocyte counts who have high 6methylmercaptopurine and low 6-thioguanine levels on thiopurine treatment. They recommend careful monitoring for leukopenia in treated patients.

Original article Sparrow MP et al. (2007) Effect of allopurinol on clinical outcomes in inflammatory bowel disease nonresponders to azathioprine or 6-mercaptopurine. Clin Gastroenterol Hepatol 5: 209-214

\section{Central venous monitoring is not required during living-donor hepatectomies}

A major concern during living-donor liver transplantation is donor blood loss and its associated morbidity and mortality. As low central venous pressure (CVP) is believed to reduce blood loss during liver resection, CVP monitoring is standard practice during living-donor hepatectomies. To see whether this rationale was supported by evidence, Niemann and colleagues investigated the effect of CVP monitoring on intraoperative management and outcomes of living-donor hepatectomies.

The authors retrospectively analyzed 50 patient records of adult-to-adult, living-donor, right-lobe hepatectomies performed at a single center in San Francisco between May 2002 and October 2005. In total, 31 patients underwent intraoperative catheterization to monitor CVP, and 19 patients were not catheterized. The presence of a central venous catheter did not result in decreased estimated blood loss or intraoperative fluid administration. There was no correlation between fluid administration and estimated blood loss in either group; this result might be because a policy of fluid restriction was applied to all patients. There were no differences in operating time, postanesthesia careunit stay, or hospital stay. No difference was observed between the groups for the frequency of complications either during hospitalization or at the 3-month follow-up.

The authors conclude that intraoperative fluid requirements and blood loss are equivalent in patients who undergo living-donor, right-lobe hepatectomies, with or without central venous catheterization. CVP monitoring might not, therefore, be necessary during right-lobe liver donation in centers with extensive experience in living-donor hepatectomies.

Original article Niemann CU et al. (2007) Central venous pressure monitoring during living right donor hepatectomy. Liver Transpl 13: 266-271

\section{Zinc carnosine prevents NSAID-induced injury and stimulates mucosal repair}

The sale of health-food supplements produces an annual turnover of $\$ 18$ billion in the US, although scientific evidence that supports their use in prevention and treatment of illness is often limited.

Playford and colleagues used in vitro and in vivo models and a clinical trial to analyze the effects of zinc carnosine on gut mucosal integrity and repair. This supplement is marketed as having beneficial effects on gastrointestinal health.

Zinc carnosine induced dose-dependent increases in cell migration and cell proliferation in in vitro assays (maximal responses were seen at concentrations of $100 \mu \mathrm{mol} / \mathrm{l}$ ) and reduced mucosal injury in well-validated rodent models of gastrointestinal damage (by $38 \%$ at $1 \mathrm{mg} / \mathrm{ml}$, and by $75 \%$ at $5 \mathrm{mg} / \mathrm{ml}$ doses). Furthermore, administration of zinc carnosine at the standard recommended dose $(37.5 \mathrm{mg}$ twice daily) abrogated NSAID-induced intestinal permeability, an indication of mucosal damage, in 10 volunteers (aged $24-40$ years, 5 men and 5 women).

The authors conclude that zinc carnosine stabilizes gut mucosa and should be classified as a cytoprotective agent, because it protects against NSAID-induced injury without altering 